\title{
Disorders of appetite and eating
}

Appetite disorders are common in most fields of medicine and take a heavy toll on the health care system

\section{Regulation of appetite}

Appetite is defined as an instinctive physical desire for food or drink, which is often felt as hunger, and serves to ensure adequate energy intake to maintain metabolism. Recent advances in physiological, biochemical, genetic and behavioural sciences have helped towards a better understanding of multiple inter-related factors concerned in the regulation of appetite and its abnormalities. As a result, the long established view that food intake is tightly controlled by hypothalamic centres is now replaced by the concept that appetite and eating are regulated by a complex, integrated network of neural and chemical signals [1]. Neural signals relaying on the hypothalamic nuclei are received from cortical, brainstem and limbic areas of the brain, and also from the oro-pharyngeal region, other parts of the gastrointestinal system and adipose tissue [2]. The mediators of these signals are both neurochemical and hormonal. They act from peripheral and central sites [1]. The central mediators are the orexigenic neuropeptides such as neuropeptide Y (NPY), Agouti related peptide (AGRP) and orexins, and the anorexigenic neuropeptides, melanocortins and cocaine and amphetamine related peptide (CART) [1]. The identified peripheral mediators include cholecystokinin (CCK), peptide YY, ghrelin, leptin and insulin. Additionally, appetite is further influenced by behaviours learnt through positive and negative conditioning to taste, which determine the choice of food [3]. For example, the 
high affinity for sweet and fatty foods despite satiety is due to reward pathways in the brain, mediated via opioids and dopamine [2]. Knowledge of this elaborate system of signals is valuable in understanding the mechanism of onset and the maintenance of appetite disorders, and their effective management.

Disorders of appetite can present as abnormally excessive or reduced food intake. The underlying causes could be biological or psychological or a combination of both [3]. Cultural and ethnic factors too are considered to be significant in the origin of some disorders of appetite [3]. Further, the reduced or excessive food intake may present in bouts as in the major eating disorders of anorexia and bulimia nervosa, or persist over a period of time such as in chronic illness or cancer.

\section{Disturbances of appetite in physiological states}

Appetite disturbances related to physiological states are a feature of both early and advanced pregnancy. In early pregnancy, the loss of appetite is associated with nausea and vomiting of pregnancy (NVP), ranging from mild to severe. NVP is the result of complex interactions between genetic, hormonal and environmental factors during the period of organogenesis [4]. Hyperphagia usually occurs in the second and third trimesters of pregnancy and during lactation. Animal studies have shown that pregnancy induced hyperphagia is related to a decrease in leptin, leading to a reduced satiety effect, up-regulation of orexigenic factors and attenuation of anorexigenic pathways[5]. Further, the hyperprolactinaemia in lactation may influence hypothalamic neuropeptide levels, leading to increased feeding [6].

Physiological anorexia of ageing and loss of weight are usually seen in people over 70 years. The anorexia is partly due to changes in taste and smell. Adaptive relaxation of the fundus of the stomach is also reduced, leading to more rapid filling and early satiety [7]. In addition, the level of the satiating hormone CCK increases with age [1]. Nutritional interventions may be beneficial in preventing weight loss but maintenance of physical activity too is essential for a good outcome [7].

\section{Disorders of appetite in physical illness}

A defective hypothalamic regulation of appetite has been identified in cachexia inducing conditions such as cancer, chronic kidney disease, chronic inflammatory conditions, AIDS, and chronic pulmonary and cardiovascular diseases [8]. However, the appetite disturbance in this cachexia-anorexia syndrome is more likely to be multifactorial, where persistent nausea and vomiting, early satiety, malabsorption and gastric stasis may contribute in different degrees to food aversion [8]. Cytokines such as interleukins and tumour necrosis factor (TNF), which have anorectic properties, play a major role in the development of cachexia by inhibiting feeding. Their action mimics the hypothalamic effect of negative feedback through inhibition of NPY [9]. This action of cytokines in wasting disorders prevents the adaptive mechanism otherwise seen in normal individuals in response to weight loss [9].

In cancer and chronic inflammatory conditions, a multidisciplinary approach to care is necessary [10]. Nutritional therapy alone is inadequate, since the causative factors of anorexia-cachexia are diverse. The therapeutic options are to increase nutritional intake and to inhibit muscle and fat wasting with a variety of pharmacological agents [10]. Progestational agents and corticosteroids are known to reduce cancer induced cachexia-anorexia [10]. They act on the appetite regulatory circuitry to increase appetite, inhibit tumour derived catabolic agents, and reduce tissue wasting and cytokine release [10]. Other drugs with similar actions are antiserotonergics and the branched-chain amino acids, eicosapentanoic acid and cannabinoids [10]. Identification and treatment of other causes of reduced food intake, such as nausea and vomiting directly related to treatment, oral mucositis, and gastrointestinal obstruction is essential. As weight loss shortens the survival time of cancer patients, effective therapy leads to improved quality of life [9].

Hyperphagia and obesity are common sequelae of tumours of the hypothalamic region and their treatment with surgery and radiotherapy [11]. Damage to the ventromedial hypothalamus leads to hyperphagia, a low metabolic rate, growth hormone $(\mathrm{GH})$ deficiency and autonomic imbalance that contribute to weight gain [11]. However, with the exception of GH replacement, few clinical trials have evaluated significant numbers of patients and their management by behavioural, dietary, pharma-cological and surgical approaches [11].

The worsening global epidemic of obesity has led to some urgency in understanding appetite regulation. Much of human obesity is multifactorial and is usually due to relative overconsumption [1]. Dietary and behavioural modification and physical exercise are the key forms of treatment for many obese individuals. Pharmacological therapy also has a role in the management of some. The National Institute for Clinical Excellence (NICE), UK, guidelines recommend drugs such as sibutramine, a serotonin, and noradrenaline re-uptake inhibitor [12]. Fenfluramine, a popular drug used for appetite suppression is now prohibited from use because of unacceptable side effects [13]. Another form of treatment for patients with morbid obesity is intestinal resection and bypass surgery, where reduced secretion of ghrelin and PYY have been implicated as contributing to weight loss [14].

\section{Disorders of appetite in psychological disturbances}

Clinically significant disturbances of appetite and eating related to psychological phenomena have been 
observed in all age groups. They closely correlate with physical and physiological changes in producing the symptoms and signs. The most widely studied disorders are bulimia nervosa and anorexia nervosa . Bulimia nervosa is characterised by episodes of binge eating of high calorie food followed by inappropriate compensatory behaviour such as self-induced vomiting $[15,16]$. It may occur in response to stressful experiences. Patients with bulimia need a larger amount of food to feel fullness during a meal, suggesting that ingested food has a reduced satiating effect [3]. Current evidence indicates that faulty negative feedback mechanisms either peripherally in the gut or centrally in the brain could be responsible [3]. Behavioural strategies are the main form of management [15].

In contrast, anorexia nervosa (AN) is a disorder with recurrent episodes of severe restriction of food intake. There is an intense fear of weight gain and a disturbance in the perception of body size $[15,16]$. Specific neuroendocrine factors are associated with the aetiology of AN. Corticotrophin releasing hormone $(\mathrm{CRH})$, a potent anorectic hormone, is increased during the illness and returns to normal following nutritional rehabilitation [3]. $\mathrm{CRH}$ is also responsible for maintenance of anorectic behaviour and initiating relapse. Conversely, serotonin, though inhibitory to feeding, is implicated in the treatment of AN. Both serotonin agonists (clomipramine, fluoxetine) and antagonists (cyproheptadine) are useful as adjunct treatments of AN [3]. Further, the role of the inhibitory influence of oestradiol on the appetite has also been investigated since AN has a higher predisposition in females and often develops near menarche [17]. There is accumulating evidence that oestradiol may decrease meal size by increasing the potency of the satiating actions of some gut peptides, especially cholecystokinin [17]. These findings suggest that there may be hope for treatment of AN through manipulation of the interaction of oestradiol with peripheral and central neural controls of eating behaviour [17]. Very low levels of leptin are seen in emaciated patients with $\mathrm{AN}$ and these improve with weight gain, indicating a possible association [18].

A variety of psychologically based appetite and eating disorders are also described in infants and children $[15,16]$. Disorders such as pica, rumination, and psychogenic failure to thrive are well documented. In pica, the characteristic feature is a persistent eating of nonnutritive substances associated with the risk of lead poisoning, helminth infections and intestinal obstruction $[15,16]$. Repeated regurgitation and re-chewing of food is seen in infants or children with rumination disorder resulting in poor growth $[15,16]$. These conditions result in marked deceleration of weight gain with disruption of emotional and social developmental milestones, and may even be life-threatening. They are known to be associated with disturbed temperament in the infant or child, negative emotional relationship with the primary caregiver or being brought up in adverse environments [19]. However, consistent physiological and endocrine disturbances have not been identified probably due to the complex nature of these conditions. Disturbance of appetite is also a prominent feature in some inherited disorders in children. Recognised syndromes include Prader-Willi, Klinefelter, Frohlich, Laurence-Moon-Biedl, Kleine-Levin and Mauriac syndromes [19].

The appetite control systems are designed to maintain a stable body weight despite great variations in daily food intake and expenditure. Over the past decade, our understanding of the systems that regulate food intake and body weight has been augmented by new research findings. Further understanding of appetite and body weight control are likely to provide novel therapeutic approaches for a range of appetite and eating disorders.

\section{References}

1. Wynne K, Stanley S, McGowan B, Bloom S. Appetite control. Journal of Endocrinology 2005; 184: 291-318.

2. Wynne K, Stanley S, Bloom S. The gut and regulation of body weight. Journal of Clinical Endocrinology and Metabolism 2004; 89: 2576-82.

3. Geary N, Smith GP. Appetite. In: Saddock BJ, Saddock VA, eds. Comprehensive Textbook of Psychiatry. 7th ed. Baltimore: Lippincott, Williams \& Wilkins, 2000: 209-18.

4. Broussard CN, Richter JE. Nausea and vomiting of pregnancy. Gastroenterology Clinics of North America 1998; 27: 123-51.

5. García MC, López M, Gualillo O, Seoane LM, Diéguez C, Señarís RM. Hypothalamic levels of NPY, MCH, and prepro-orexin mRNA during pregnancy and lactation in the rat: role of prolactin. The Federation of American Societies for Experimental Biology Journal 2003; 17: 1392-1400.

6. Johnstone LE, Higuchi T. Food intake and leptin during pregnancy and lactation. Progress in Brain Research 2001; 133: $215-27$.

7. Wilson MM, Morley JE. Invited review: aging and energy balance. Journal of Applied Physiology 2003; 95: 172836.

8. Plata-Salaman CR. Central nervous system mechanisms contributing to the cachexia-anorexia syndrome. Nutrition 2000; 16: 1009-12.

9. Inui A. Cancer anorexia-cachexia syndrome: are neuropeptides the key? Cancer Research 1999; 59: 4493-501.

10. Inui A. Cancer anorexia-cachexia syndrome. Current issues in research and management. CA: A Cancer Journal for Clinicians 2002; 52: 72-91.

11. Pinkney J, Wilding J, Williams G and MacFarlane I. Hypothalamic obesity in humans: what do we know and what can be done? Obesity Reviews 2002; 3: 27-34.

12. National Institute for Clinical Excellence. Guidance on 
the use of sibutramine for the treatment of obesity in adults. Technology Appraisal Guidance No. 31. Issue date: October 2001. ref: N0035. At: http://www.nice.org.uk.

13. Hopkins PN, Polukoff GI. Risk of valvular heart disease associated with use of fenfluramine. BMC Cardiovascular Disorders 2003; 3: 5 .

14. Cummings DE, Weigle DS, Frayo RS, Breen PA, Ma MK, Dellinger EP, Purnell JO. Plasma ghrelin levels after dietinduced weight loss or gastric bypass surgery. New England Journal Medicine 2002; 346: 1623-30.

15. American Psychiatric Association. Diagnostic and Statistical Manual of Mental Disorders. 4th ed. Washington DC. American Psychiatric Association, 1994.
16. International Classification of Mental and Behavioural Disorders. 10th ed. Geneva: World Health Organization, 1992.

17. Geary N. The Effect of Estrogen on Appetite. Medscape General Medicine 1(3), 1999. At: http:// www.medscape.com/viewarticle/408893.

18. Eckert ED, Pomeroy C, Raymond N, Kohler P, Thuras P, Boners CY. Leptin in anorexia nervosa. Journal of Clinical Endocrinology and Metabolism 1998; 83: 791-5.

19. Woolston JL. Eating and growth disorders in infants and children. In: M. Lewis, ed. Child and Adolescent Psychiatry-AComprehensive Textbook. 3rded. Baltimore: Lippincott, Williams \& Wilkins, 2002: 681-92.

Hemamali Perera, Department of Psychological Medicine, and Angela de Silva, Department of Physiology, Faculty of Medicine, University of Colombo.

Correspondence: AdeS, e-mail: <ang2sl@yahoo.com>. (Competing interests: none declared).

\section{Subscription Rates}

Why should you buy the Ceylon Medical Journal regularly, and submit your article to it? Because it is listed in the Index Medicus, BIOSIS, CAB INTERNATIONAL and EMBASE, articles get wide publicity. Because the $C M J$ conforms to the International Council of Medical Journal Editors' criteria, its editing is of a very high standard, and its articles are of good scientific quality. We are hopeful of getting listing by Science Citation Index soon. And the $C M J$ is the oldest surviving English medical journal in the whole of Australasia.

Annual subscription rates for residents overseas who are not members of the Sri Lanka Medical Association, and not Sri Lankan citizens, are as follows.

$\begin{array}{llll}- & \text { SAARC countries } & \text { USD } & 20 \\ - & \text { UK and Europe } & \text { USD } & 30 \\ & \text { All other countries } & \text { USD } & 35\end{array}$

Editors, Ceylon Medical Journal, Wijerama House, 6, Wijerama Mawatha, Colombo 7, Sri Lanka. Inquiries: e-mails: <hjdes@sltnet.lk> or <colvin_goonaratna@yahoo.com>.

(Subscription form on page 31) 